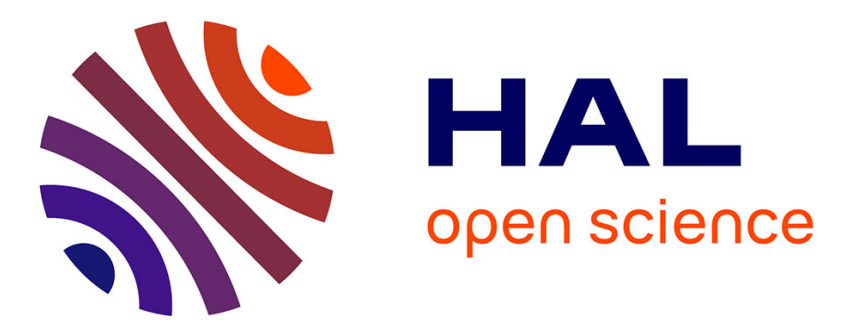

\title{
Optimized Handover and Resource Management: An 802.21 Based Scheme to Optimize Handover and Resource Management in Hybrid Satellite-Terrestrial Networks
}

\author{
Riadh Dhaou, Ryad Ben-El-Kezadri, Julien Fasson, Fabrice Arnal, Emmanuel \\ Dubois, Patrick Gelard
}

\section{To cite this version:}

Riadh Dhaou, Ryad Ben-El-Kezadri, Julien Fasson, Fabrice Arnal, Emmanuel Dubois, et al.. Optimized Handover and Resource Management: An 802.21 Based Scheme to Optimize Handover and Resource Management in Hybrid Satellite-Terrestrial Networks. International Journal of Satellite Communications and Networking, 2014, vol. 32 ( $\left.\mathrm{n}^{\circ} 1\right)$, pp. 1-23. 10.1002/sat.1054 . hal-01123775

\section{HAL Id: hal-01123775 \\ https://hal.science/hal-01123775}

Submitted on 5 Mar 2015

HAL is a multi-disciplinary open access archive for the deposit and dissemination of scientific research documents, whether they are published or not. The documents may come from teaching and research institutions in France or abroad, or from public or private research centers.
L'archive ouverte pluridisciplinaire HAL, est destinée au dépôt et à la diffusion de documents scientifiques de niveau recherche, publiés ou non, émanant des établissements d'enseignement et de recherche français ou étrangers, des laboratoires publics ou privés. 


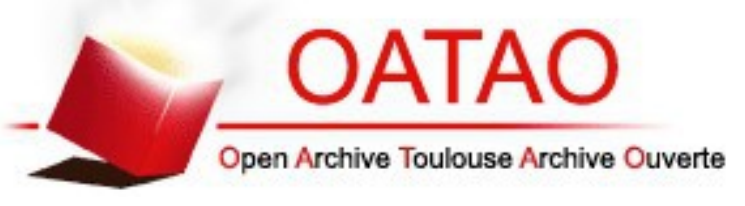

\section{Open Archive TOULOUSE Archive Ouverte (OATAO)}

OATAO is an open access repository that collects the work of Toulouse researchers and makes it freely available over the web where possible.

This is an author-deposited version published in : http://oatao.univ-toulouse.fr/ Eprints ID : 12665

To link to this article : DOI :10.1002/sat.1054

URL : http://dx.doi.org/10.1002/sat.1054

To cite this version : Dhaou, Riadh and Ben-El-Kezadri, Ryad and Fasson, Julien and Arnal, Fabrice and Dubois, Emmanuel and Gelard, Patrick Optimized Handover and Resource Management: An 802.21 Based Scheme to Optimize Handover and Resource Management in Hybrid Satellite-Terrestrial Networks. (2014) International Journal of Satellite Communications and Networking, vol. 32 ( $\left.\mathrm{n}^{\circ} 1\right)$. pp. 1-23. ISSN 1542-0973

Any correspondance concerning this service should be sent to the repository administrator: staff-oatao@listes-diff.inp-toulouse.fr 


\title{
Optimized handover and resource management: an 802.21-based scheme to optimize handover and resource management in hybrid satellite-terrestrial networks
}

\author{
Riadh Dhaou $^{1, *, \dagger}$, Ryad Ben-El-Kezadri ${ }^{1,2}$, Julien Fasson ${ }^{1}$, Fabrice Arnal ${ }^{3}$, \\ Emmanuel Dubois ${ }^{4}$ and Patrick Gelard ${ }^{4}$ \\ ${ }^{1}$ INPT-ENSEEIHT/IRIT, 2 rue Charles Camichel BP7122, F-31071 Toulouse Cedex 7, France \\ ${ }^{2}$ CRISTAL, University of Manouba 2010, Tunisia \\ ${ }^{3}$ Thales Alenia Space, 26 avenue JF Champollion, BP 1187, 31037 Toulouse Cedex, France \\ ${ }^{4}$ Centre National d'Etudes Spatiales, 18 avenue Edouard Belin, 31401 Toulouse Cedex 9, France
}

\section{SUMMARY}

Satellite communications can provide fourth generation (4G) networks with large-scale coverage. However, their integration to $4 \mathrm{G}$ is challenging because satellite networks have not been designed with handover in mind. The setup of satellite links takes time, and so, handovers must be anticipated long before. This paper proposes a generic scheme based on the Institute of Electrical and Electronics Engineers 802.21 standard to optimize handover and resource management in hybrid satellite-terrestrial networks. Our solution, namely optimized handover and resource management (OHRM), uses the terrestrial interface to prepare handover, which greatly speeds up the establishment of the satellite link. We propose two mechanisms to minimize the waste of bandwidth due to wrong handover predictions. First, we leverage the support of 802.21 in the terrestrial access network to shorten the path of the signaling messages towards the satellite resource manager. Second, we cancel the restoration of the satellite resources when the terrestrial link rolls back. We use OHRM to interconnect a digital video broadcasting and a wireless $4 \mathrm{G}$ terrestrial network. However for the simulation tool, we use a WiMAX as the terrestrial technology to illustrate the schemes. The simulation results show that OHRM minimizes the handover delay and the signaling overhead in the terrestrial and satellite networks.

\section{INTRODUCTION}

Fourth generation $(4 \mathrm{G})$ networks are expected to ensure seamless communication between heterogeneous technologies and to offer personalized services to users and to increase to increase highly the bandwidth available to user. $4 \mathrm{G}$ represents a win-win opportunity for terrestrial and satellite Internet service providers (ISP) to increase profits. By setting roaming agreements with satellite providers, terrestrial ISPs can benefit from the long-range of satellite communications (satcoms) to extend their network coverage. In return, the satellite providers can leverage the low-cost infrastructures of terrestrial ISPs to lower their charge. The main application of hybrid satellite-terrestrial networks (HSTNs) is the support of real time services in highly mobile scenarios. This includes telephony and broadband Internet access in collective terminal environments like trains, ships, and road vehicles. In emergency response operations and battlefield conditions, HSTNs can support voice and video services for

\footnotetext{
*Correspondence to: Riadh Dhaou, IRIT-ENSEEIHT, 2 rue Camichel BP7122, F-31071 Toulouse Cedex 7, France

†E-mail: riadh.dhaou@enseeiht.fr
} 
mobile units. They can also provide permanent network connections for critical applications. This includes human to machine communications such as mobile robotic telesurgery and control of unmanned vehicles.

The integration of satcom in $4 \mathrm{G}$ networks is challenging because satellite networks have not been designed with hybrid handover in mind. In a stand-alone satellite system, handovers can be predicted easily. Furthermore, the time to anticipate a handover between the beams of a satellite is much lower because the mobile nodes (MN) stay connected to the same satellite. In a 4G HSTN, mobile users are continuously switching back and forth between the terrestrial and satellite systems (under the assumption that at least one link is always available). To take advantage of the terrestrial infrastructure, the satellite manager has to release the resources allocated to an MN each time the MN connects to the terrestrial network. When the MN comes back to the satellite network, the allocation of the satellite resources must be anticipated long in advance because the latency of the satellite link delays the resource request and the actual delivery of these resources. This makes the handover prediction particularly uncertain especially when the quality of the terrestrial signal varies. Incorrect predictions can lead to important waste of satellite bandwidth.

In this paper, we show the relevance of the Institute of Electrical and Electronics Engineers 802.21 standard [1]—also known as media independent handover (MIH) — to design a 4G internet protocol (IP)-based HSTN. The goal of the MIH function (MIHF) is to facilitate handover between heterogeneous networks. It enables the higher layers to control the communication links and to monitor their status. The MIHF is distributed all across the network (within the MNs and the terrestrial base stations) and uses specific messages to transport data.

Our contribution is the design and the evaluation of a scheme, called optimized handover and resource management (OHRM), that optimizes handover and resource management in 4G HSTNs. It is motivated by four observations: (i) MIH is well suited to HSTNs because it proposes a generic interface to manage level 2 events. Consequently, it allows to anticipate vertical handover events and to cancel them if the link rolls back. We show the flexibility of our scheme through different strategies for monitoring links; (ii) the satellite manager needs to monitor the arrival and the departure times of the MNs in the satellite network to (de)allocate resources. It is actually possible to get this information from the times when the MNs enter and leave the terrestrial network. We assume that there is no discontinuity between satellite and terrestrial networks. Indeed, the proposal aims at optimizing the use of satellite resources and not really focusing on seamless handover. This is because a departure (respectively an arrival) from the terrestrial network basically corresponds, under our assumption, to an arrival (respectively a departure) in the satellite network. In other words, the arrivals and departures in the satellite network can be reported to the satellite manager without monitoring the satellite interface. Actually, monitoring the satellite interface has limited interest because the cause of the up handovers ${ }^{\ddagger}$ (which are the main challenges in HSTNs) is the departure from the terrestrial network. Monitoring terrestrial links only has the advantage to simplify the product design: an OHRM terminal needs a terrestrial chip with MIH capabilities, whereas the satellite interface can be kept unchanged. Furthermore, the status of the terrestrial link is naturally sent through the terrestrial interface, which is must faster than the satellite one; (iii) the terrestrial link can be monitored by the $\mathrm{MN}$ or the point of attachment $(\mathrm{PoA})$ in the access network. PoAs are much convenient for this task because they are connected by cable to the satellite ground network. MIH allows the delegation of the monitoring task to the PoAs without requiring the installation of new softwares in the network; and (iv) handovers in HSTNs fundamentally differ from classical vertical handovers because the MNs are ultimately expected to return to the satellite network. As the satellite network is generally used as last resort, when no other network is available, the resources of the MNs have to be recovered when the mobiles switch back to their satellite interface. To this purpose, OHRM introduces the concept of virtual satellite terminals. The virtual terminals are the satellite terminals that are connected to the terrestrial network. They are managed as standard satellite terminals, but their resources are assigned to other active satellite connections. The satellite resources are returned to the MNs when they come back to the satellite network. OHRM estimates the bandwidth of the terrestrial connection, re-enables the

\footnotetext{
Thandovers from the terrestrial to the satellite
} 
satellite link, and transfers the communication without the assistance of the MN in order to speed up the start-up of the satellite allocation loop.

To validate the proposed scheme, we integrate OHRM in the NS-2 simulator and illustrate it through a hybrid digital video broadcasting return channel via satellite (DVB-RCS(2))/WiMAX network. This last technology was selected rather than long term evolution (LTE) for simulation convenience, but our conclusions will remain valid for any other $4 \mathrm{G}$ wireless technology. The simulation results show that OHRM jointly optimizes the handover delay and the bandwidth of the networks when the PoA monitors the link.

The remainder of the paper is organized as follows. Section 2 presents the state of the art. Sections 3 and 4 describe the architecture and the functioning of OHRM. The simulation platform is detailed in Section 5. Section 6 compares the performance of OHRM depending on whether monitoring is performed by the MN or the PoA. Section 7 discusses important considerations regarding the implementation of OHRM in real networks. Section 8 contains concluding remarks.

\section{RELATED WORK}

The first generation of networks combining satellite and terrestrial components is characterized by a high asymmetry. The broadcast capacity of satellites is used on the downlink whereas the low bandwidth terrestrial network for the uplink. In recent years, new generations of HSTN have been designed [2, 3], and several international initiatives have been launched to classify the emerging satcom architectures. The European Telecommunications Standards Institute proposes three classes of systems combining satellite and terrestrial components: concatenated, multi-radio access technology (RAT), and hybrid [4]. The first class defines an interconnection of a terrestrial access to a satellite backhaul, or vice versa. The second class refers to a system where the end user device implements independent terrestrial and satellite RATs. A system that is neither concatenated nor multi-RAT is considered as hybrid. Along the same lines, the integrated satellite initiative proposes a typology based on service delivery and spectrum usage [5]. The level of integration of a combined system are themselves defined by the ITU-R [6]. This integration aims at reducing network complexity and providing better mobility support for users. In this paper, as in most of the literature,the terms hybrid and multi-RAT (as per the European Telecommunications Standards Institute definition) are used interchangeably.

The main research driving factor for hybrid wireless networks is seamless handover. Two types of handovers can be defined according the nature of the integrated system:

- Horizontal handover refers to the case in which the architecture is based on the extension of the terrestrial radio access network (T-RAN) standards to the S-RAN (satellite RAN), or vice versa. Such extensions include the universal mobile telecommunications system (UMTS) to S-UMTS, DVB-handheld to DVB-satellite services to handhelds, T-digital multimedia broadcasting (T-DMB) to SDMB/MAESTRO and LTE to S-LTE. Lightsquared 4G-LTE-satellite network is an example of such architecture [7]. These integrated systems are mainly designed for a geographic extension of the services provided by the hybrid RAN. The handover process can be optimized on the basis of each standard's own service primitives. The integration and the handover schemes are specific to these architectures. Some standard's organizations (such as 3rd generation partnership project (3GPP/3GPP2)) have defined two handover modes: optimized and nonoptimized. The only standardized handover solutions are based on a non-optimized mode. For instance, the GEO-Mobile Radio (GMR) standard does not define any optimized mode.

- Vertical handover refers to the case in which the architecture combines heterogeneous technologies (DVB-RCS(2) with LTE or with Institute of Electrical and Electronics Engineers standards as we propose in [8] and in this paper). Handover management can rely either on specific optimized solutions or on standard generic solutions. Specific solutions may be optimized for each couple of technologies, but they lack flexibility. A generic standard solution, such as MIH, can evolve more naturally and easily in order to integrate new technologies. 
The MIH standard offers an effective way to manage handovers between heterogeneous link layer technologies. Open source implementations $[9,10]$ and simulation models $[11]$ are now available for most wireless technologies. MIH solutions have also been proposed for DVB [12] and HSTN systems [13-15]. Qureshi and Dadej [14], in particular, present the challenges related to the support of MIH in satellite interfaces, such as the mapping of the MIH primitives to the S-RAN. The limitations of these works is that they only focus on handover management and on optimization of upper layers (network, transport, and session). They do not consider the trade-off between the user requirements, in terms of quality of service, during handover, and the operators requirements, in terms of optimization of the resource usage.

This paper not only takes into account the handover performance but also the operator's requirements. In an HSTN, the allocation of the satellite and terrestrial resources may be centralized or distributed. The centralized solution relies on a global resource manager. Because this latter acts as a central coordination entity [16], it can provide more efficient network usage and better flexibility (especially for the physical layer). However, in heterogeneous environments involving different network operators, a central entity may not be possible. OHRM represents in this context an effective way to integrate terrestrial and satellite systems.

\section{ARCHITECTURE}

\subsection{Network architecture}

Optimized handover and resource management manages satellite resources in HSTNs during up and down handovers. An up handover happens when a MN switches from the terrestrial to the satellite network. A down handover is the inverse operation. As Figure 1 shows, the terrestrial ISPs can support all the wireless technologies that implement MIH: UMTS, Wi-Fi, WiMAX, etc. The MNs include a satellite network interface card (NIC) and one or more wireless NICs. In this work, we apply OHRM to a DVB-RCS(2)-based satellite network [17]. A DVB-RCS(2) ground network includes a network control center (NCC) that notably controls the resource in the network and generates the signaling, one or multiple gateway(s) that centralizes the satcom traffic. The static resources (constant rate allocation, i.e., constant rate assignment (CRA)) are assigned at terminal $\log$ on. The dynamic resources (rate and volume-based dynamic capacity, i.e., RBDC/VBDC) are allocated on demand, according to a demand assigned multiple access (DAMA) scheme. The NCC also manages the handovers of the MNs between the different satellites and the different beams of a satellite. OHRM focuses on the allocation of user

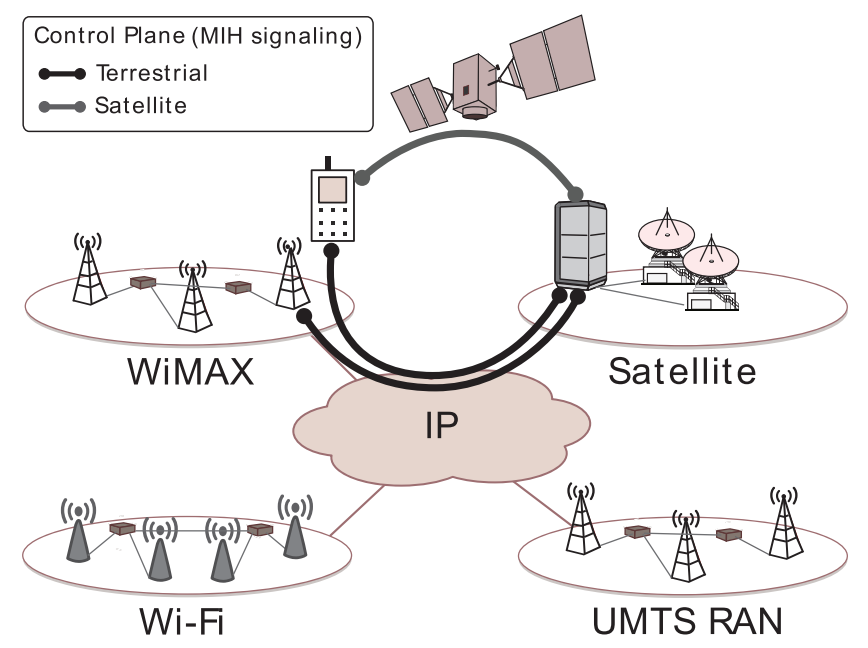

Figure 1. Architecture of a hybrid satellite-terrestrial network. 


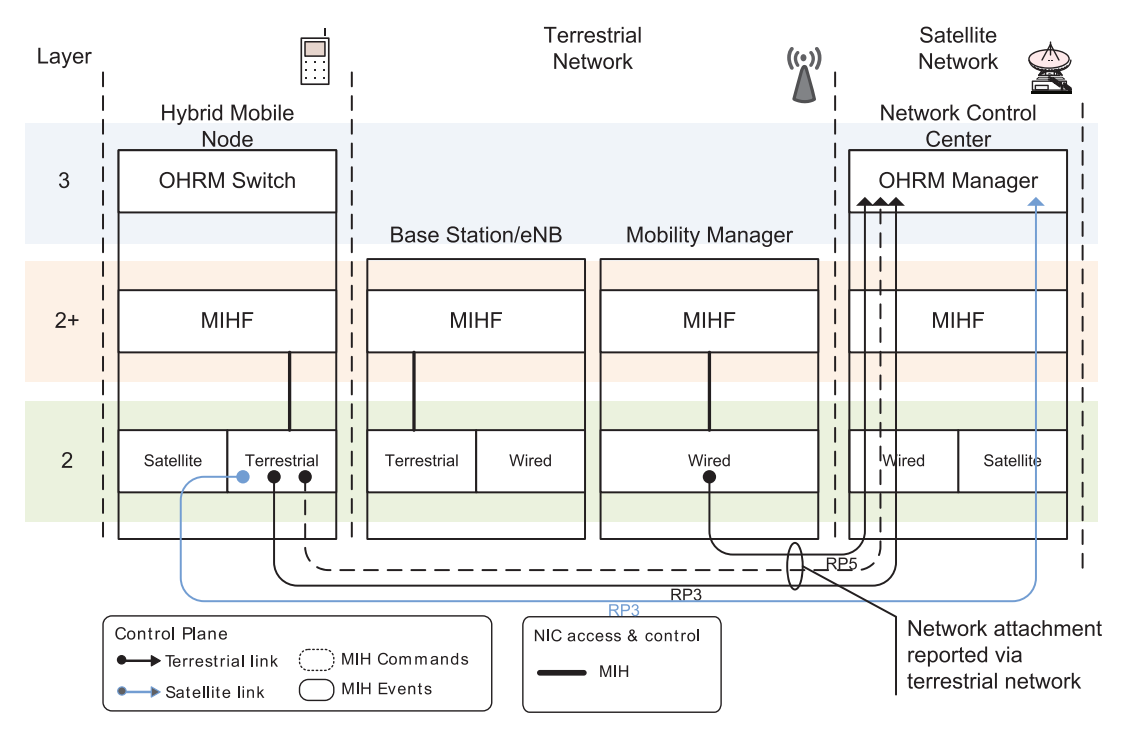

Figure 2. Optimized handover and resource management architecture.

resources on the uplink channel, ${ }^{\S}$ which is the most sensitive mechanism in satcoms because of the latency of the allocation loop. It is worth noting that the DVB technology could be interchanged with S-LTE. This is because our model is very general and suits any DAMA architecture. In an S-LTE network, the resource manager (which is hosted in the NCC in DVB-RCS(2)) would be included in the packet data network gateway, and the traffic would be carried by guaranteed bit rate (GBR) and non-GBR bearers instead of CRA, RBDC, and VBDC slots.

The basic vertical handover scenario is the following. We consider a global area of service covered by a multi-beam DVB-RCS(2)+M satellite network and completed by a 4G terrestrial coverage. The access technology selection is based on collaboration between terminal and network; in our implementation, the decision is taken at NCC, on the basis of the signaling analysis.

The 4G terrestrial network is selected each time the connection is available, because of preferred QoS and cost. We assumed that the selection only depends on the user terminal location. When this connection becomes unavailable, the terminal switches to the DVB-RCS(2)+M network. Meanwhile, the terrestrial interface continues to scan PoA availability. When a new PoA is finally discovered, the terminal connects to it and diverts its traffic back to this interface. However, the DVB-RCS(2)+M logging is kept while continuing receiving/sending forward link/return link synchronization.

\subsection{Optimized handover and resource management architecture}

Optimized handover and resource management uses $\mathrm{MIH}$ as a middleware to communicate and to access the NICs. An MIH-capable NIC can be configured and monitored through the MIHF. MIHF provides event and command services to the MIH applications. The MIH event service (MIES) allows an application to subscribe to the events issued by a local or remote NIC. The MIH command service (MICS) allows the applications to send local and remote commands to configure an NIC or to communicate with a peer.

Figure 2 shows OHRM architecture. The MIHF is installed in the MN, the NCC, and the PoA. The advantages of $\mathrm{MIH}$ as a middleware are the wide support of 802.21 in the network equipment and the possibility to delegate some functions in the PoAs. Thanks to MIH, the development of OHRM is limited to the application level at the MN and NCC sides.

\footnotetext{
$\S_{\text {i.e., the return link in the DVB-RCS(2) terminology }}$
} 
The application in the NCC is called the OHRM manager. It controls the resources of the virtual satellite users. The OHRM switch runs in the MN. It redirects the data flows between the NICs during handovers and notifies the OHRM manager when a terrestrial link is activated or lost. The OHRM switch can act on behalf of the OHRM manager. For instance, it can command the local MIHF to forward specific MIH events to the NCC. The OHRM switch also subscribes to local events for itself because it needs the status of the terrestrial radios to perform a handover. Figure 2 shows how the status of the terrestrial link is reported to the NCC. Link activations are reported with MIH commands and link losses with events. Most of the notifications are sent through the terrestrial link. The satellite link is only used after an up handover to confirm that the terrestrial link has been lost. This is not a limitation because these confirmations tolerate delay. As Figure 2 shows, the monitoring of the link losses can be delegated to the PoA.

In the MIH terminology, the signaling interfaces that connect the NCC to the MN and the PoA are respectively called reference point 3 (RP3) and RP5. OHRM does not require the satellite NICs in the $\mathrm{MN}$ and the NCC to support MIH. However, MIH could replace the driver of the NIC for certain operations. For instance, under DVB-RCS(2), the OHRM switch may use MIH to know if the log on procedure has been passed.

\section{THE OPTIMIZED HANDOVER AND RESOURCE MANAGEMENT SCHEME}

Optimized handover and resource management does not interfere with the command sequences proposed in the standard. It just adds event messages to monitor links. As a result, the decision and the execution of a handover comply with the MIH guidelines, and OHRM supports all the handover strategies proposed in the standard (mobile-controlled, mobile-initiated, network-controlled, and network-initiated handover strategies). The choice of the strategy depends on the ISP of the user, the roaming agreement, and the user contract, which are beyond the scope of this work.

The richness of the MIES and MICS services offers the applications a lot of flexibility to control handovers. This section explains how OHRM uses MIH to

(1) report the link status to the NCC,

(2) delegate the monitoring task to PoAs, and

(3) manage the resources of the satellite network.

\subsection{Link monitoring strategies}

Optimized handover and resource management only monitors terrestrial links. This is not restrictive because the arrival and departure times from the satellite network can be deduced from the entries and exits of the terrestrial network. Actually, it considerably improves the reactivity of the application because it is much faster to report the status of the connection through the air interface rather than the satellite channel. The monitoring function notifies the NCC about the activation and loss of links. Different variants of this function can be implemented. The reactive strategy alerts the NCC when it loses the signal. The predictive mode notifies the NCC when the signal starts to fade. The links can also be monitored with periodic HELLO messages as in routing protocols. OHRM implements a predictive strategy because resources can not be granted instantly in satellite systems, and hence, handovers must be anticipated.

4.1.1. Link loss. In the predictive strategy, a link alert is triggered when the power of the signal falls below a certain threshold. Alerts are reported to the NCC with MIH_Link_Parameters_Report messages. The messages carry an indication about the thresholds that have been crossed, and the applications filter them accordingly. Different thresholds are used by the switch and the manager to dissociate the times when to perform the redirection of the flows in the $\mathrm{MN}$ and the resource allocation in the NCC. For satcom in particular, the allocation must be anticipated very early because 


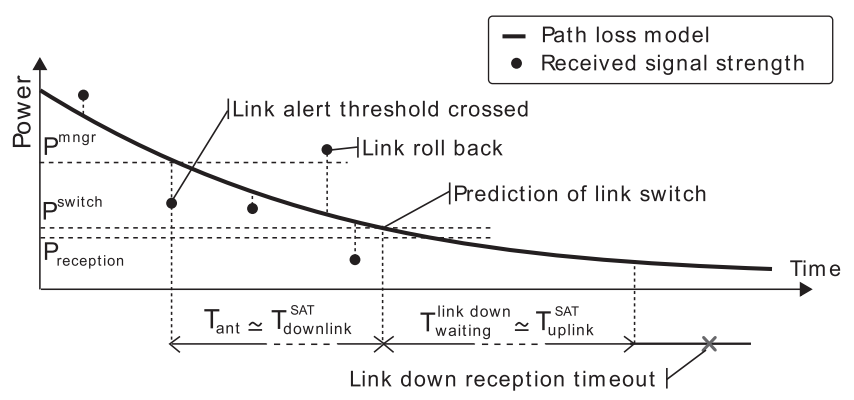

Figure 3. Trigger thresholds of optimized handover and resource management events.

the activation of the links takes a long time. The anticipation interval, $T_{a n t}$, can be computed from the latency of the RP3 path" and the satellite downlink" as given in equation 1:

$$
T_{\text {ant }}=T_{R P}+T_{\text {downlink }}^{\text {SAT }}
$$

$T_{R P}$ represents the time to report a link alert to the NCC via the terrestrial channel. $T_{\text {downlink }}^{S A T}$ is the latency of the geostationary satellite link (about a quarter of a second). It accounts for the propagation time of the uplink allocation map from the NCC to the MN via the satellite channel. It is the predominant term of equation 1.

Figure 3 shows the decrease of the power of the terrestrial signal when the MN is moving out of the coverage of the terrestrial network. The solid line corresponds to the prediction of a propagation model and the dots to the power actually received. The measures do not exactly match the model because the trajectory of the mobile and the conditions of reception are varying. The power threshold required to generate the link alerts $T_{a n t}$ before the signal is actually lost is denoted by $P^{m n g r}$. Its value depends on the anticipation interval, the speed of the mobile, and the path loss exponent of the propagation model [18].

The MIH_Link_Down event is generated when the link connectivity is lost. It is generally triggered at the expiration of a timeout due to a packet loss. In practice, this means that the event is generated long after the signal falls below the reception threshold $P_{\text {reception }}$. Actually, most implementations would willingly break the link and fake a link down as soon as the threshold that commands the redirection of the flows, $P^{\text {switch }}$, is crossed in order to speed up the transmission of the link down event. $P^{\text {switch }}$ must be greater than $P_{\text {reception }}$ to avoid packet loss but lower than $P^{m n g r}$ to redirect the flows after the restoration of the satellite resources:

$$
P_{\text {reception }}<P^{\text {switch }}<P^{\text {mngr }}
$$

Actually, $P^{m n g r}$ can be automatically computed from $P^{\text {switch }}$ to trigger the reallocation $T_{\text {ant }}$ before the redirection of the flows. We denote by $v$ and $\alpha$ the speed of the $\mathrm{MN}$ and the angle of the velocity vector with the tangent of the cell at the exit of the terrestrial network. $d^{\text {switch }}$ and $d^{\text {mngr }}$ are the distances from the center of the cell at which $P^{\text {switch }}$ and $P^{m n g r}$ are triggered.** We show that:

$$
\begin{array}{r}
d^{m n g r}=\sqrt{d^{\text {switch }}{ }^{2}-2 d^{\text {switch }} v T_{\text {ant }} \sin (\alpha)+\left(v T_{\text {ant }}\right)^{2}} \\
\forall v \in R^{+}, \alpha \in\left[0, \frac{\pi}{2}\right] \mid v T_{\text {ant }}<\sin (\alpha) d^{\text {switch }}
\end{array}
$$

This model holds for a collective terminal moving along a linear trajectory near the border of the cell. The condition of equation 3 guarantees that $d^{\text {mngr }}<d^{\text {switch }}$ (or equivalently that $P^{\text {switch }}<P^{\text {mngr }}$ ).

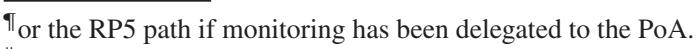

$\|_{i . e .,}$ the forward link in the DVB-RCS(2) terminology.

**i.e., $d^{\text {switch }}=\overline{P_{r}^{-1}}\left(P^{\text {switch }}\right)$ and $d^{m n g r}=\overline{P_{r}^{-1}}\left(P^{m n g r}\right)$ where $\overline{P_{r}(d)}$ is the mean received power at distance $d$ and $\overline{P_{r}^{-1}}$ its inverse function.
} 


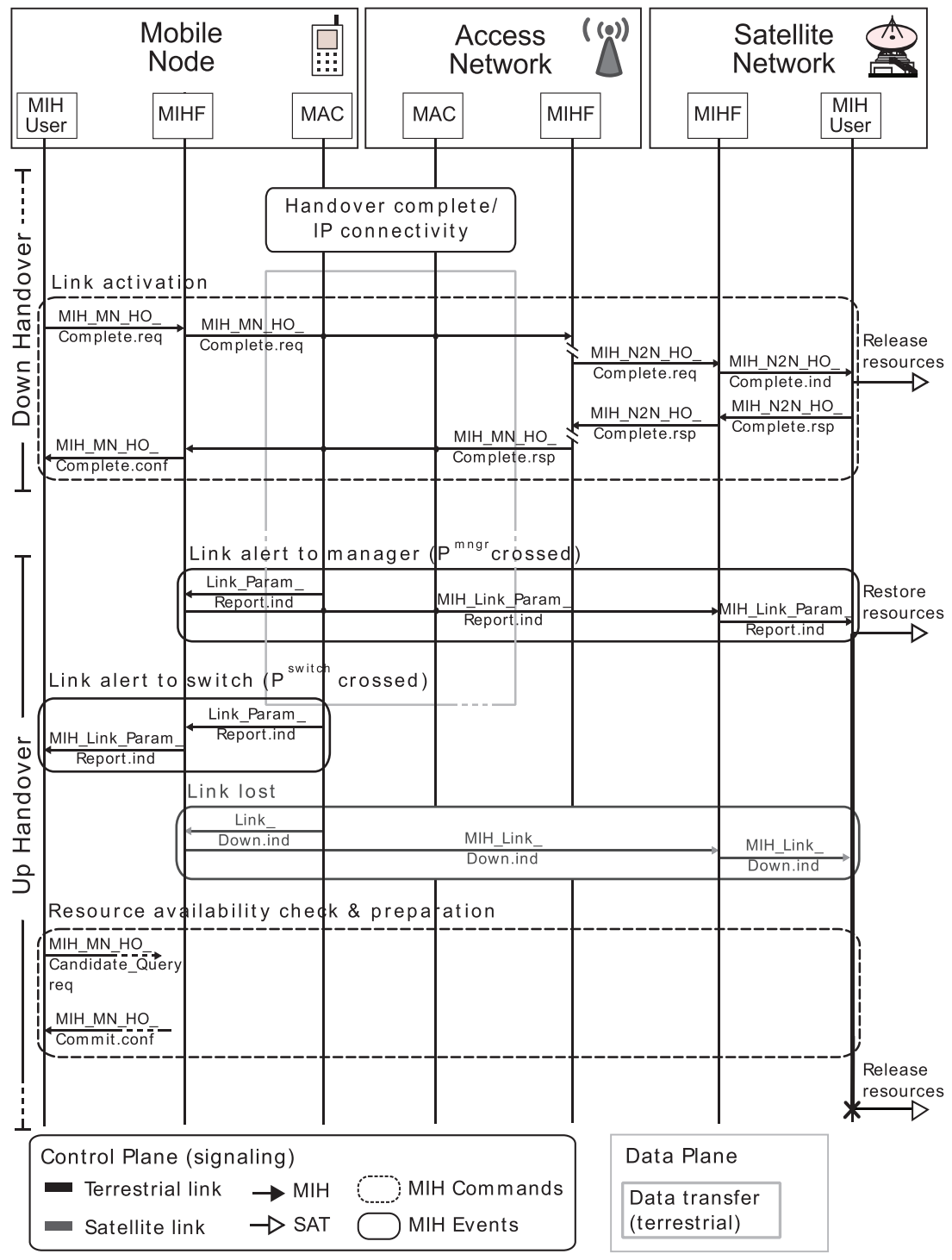

Figure 4. Optimized handover and resource management signaling when the link is monitored by the mobile nodes $(\mathrm{MN})$.

The link down event is sent via the satellite interface because the terrestrial connection is no more usable at that time. ${ }^{\dagger \dagger}$ It is received by the NCC after a delay $T_{\text {uplink }}^{S A T}$ that is about a quarter of a second later. The NCC considers the link to have rolled back if the link alerts are not followed by a link down. In this case, the NCC cancels the restoration of the resources. In the example of Figure 3, the link rolls back because the $\mathrm{MN}$ returns within the coverage of the terrestrial network. The problem is that the NCC only realizes it at the expiration of the link down timeout, which is very long because it has to account for $T_{\text {uplink }}^{S A T}$. All things considered, a roll back can use up $T_{d o w n l i n k}^{S A T}+T_{\text {uplink }}^{S A T}$ of satellite resources, which amounts to almost a second. To prevent link roll backs to waste satellite bandwidth, a roll back event can be sent to the NCC to immediately cancel the restoration of the resources if the signal gets back.

\footnotetext{
${ }^{\dagger}$ MIH_Link_Down is the only message sent over the satellite interface.
} 
4.1.2. Link activation. Link activations are reported to the NCC via the HO_Complete sequence of commands. The sequence is initiated by the MN or the PoA after an L2 or L3 handover. Figure 4 shows an example in which the sequence is initiated by the MN. The request of the MN (MIH_MN_HO_Complete.req) is re-encapsulated into a network to network handover complete request (MIH_N2N_HO_Complete.req) by the default MIH application in the PoA and forwarded to the NCC. In both cases, it is worth noting that OHRM does not alter the role of the HO_Complete commands which are primarily used to release the resources in the old network.

The activation of the terrestrial link is reported to the NCC at the end of the down handover with an MIH_MN_HO_Complete request. The message indicates that the flows have been redirected to the terrestrial network and that the MN no longer needs the satellite connection. Upon the reception of the HO Complete request, the OHRM manager switches the MN in virtual mode to reassign its resources to other satellite connections.

The lower part of Figure 4 details the up handover. In this example, the MN is initially configured (by the OHRM switch) to send link alerts to the NCC. The generation of the notifications begins when the signal level falls below $P^{m n g r}$. Upon the reception of the MIH_Link_Parameters_Report, the NCC sets the MN in satellite mode, reallocates its resources, and starts the link down timer. A second link alert is sent to the OHRM switch when the switch threshold, $P^{\text {switch }}$, is crossed. This triggers the up handover procedure and the redirection of the data flows. The alert is immediately followed by an MIH_Link_Down indication (Section 4.1.1). The NCC releases the resources of the MN if it does not receive this indication before the expiration of the link down timeout. The MN starts to check the availability of the resources in the new network with an MIH_MN_HO_Candidate_Query request as described in the MIH standard. This check always succeeds because OHRM guarantees that the satellite resources are returned to the MNs. The rest of the procedure follows the standard guidelines. It is not detailed in this paper.

\subsection{Localization of the monitoring task}

The monitoring task can be performed by the MN or the PoA. The advantage of the PoAs is to be connected by cable to the ground satellite network. Consequently, they do not consume radio resources and have shorter and more reliable access to the NCC. When monitoring is delegated to the PoA, the link alerts for the NCC flow on the RP5 path. The corresponding procedure is depicted in Figure 5. It also starts with an HO_Complete sequence. However, in this case, the OHRM manager cancels monitoring in the MN with an MIH_Event_Unsubscribe.req command and subscribes to the PoA instead. The subscription request configures the generation of the alerts with the manager's threshold, $P^{m n g r}$. The $\mathrm{MN}$ is informed that monitoring is delegated to the PoA when it receives the unsubscription request of the NCC. The OHRM switch subscribes to the PoA alerts with its own threshold, $P^{\text {switch }}$ The rest of the procedure is the same than when monitoring is performed by the MN.

\subsection{Management of satellite resources}

When the MN performs a down handover, OHRM reassigns its guaranteed resources (CRA and RBDC) to free capacity assignment (FCA) and marks the terminal as virtual. ${ }^{\S \S}$ In DVB-RCS(2) system, the use of FCA is not well defined and most of systems avoid its use. In this work, we propose to use FCA to optimize the satellite resource management. FCA stands here for capacity that can be reallocated to its initial flow at any time, it enables to assigne resource to terminals from capacity that would be otherwise unused. The resources of virtual terminals are reassigned to active connections until the MNs return to the satellite network.

\footnotetext{
$\$$ The MN and the NCC will unsubscribe to the PoA events shortly before or after the up handover.

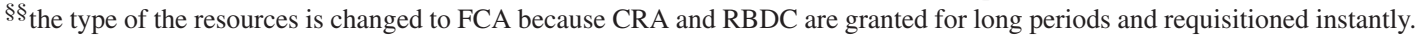




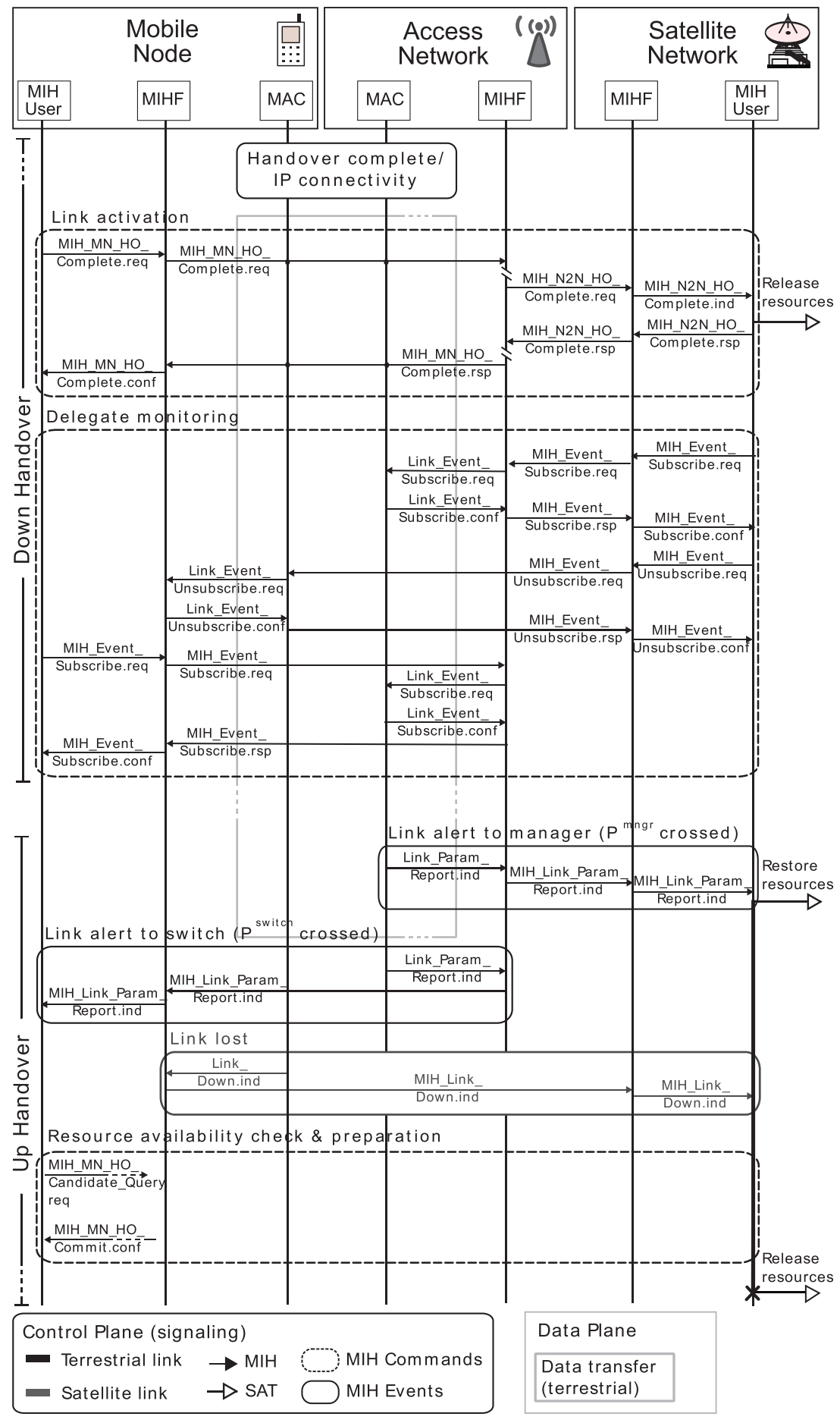

Figure 5. Optimized handover and resource management signaling when the link is monitored by the point of attachment.

4.3.1. Management of the satellite interface. After a down handover, the MN can maintain satellite synchronization or turn off its connection. The up handover process differs in the two cases.

- In the first case, the entry procedure in the satellite network is skipped, which makes the handover smoother. However, it requires the interface to be always powered up. 
- In the second case, the MN will require a new log on. The OHRM manager may alternatively allow the MN to reassociate directly via the terrestrial interface. In any case, the interface has to be turned on from time to time for the NCC to locate the virtual terminals and to manage their handovers within the satellite network. IT $^{\text {T }}$

Optimized handover and resource management anticipates handover by using the terrestrial channel. It coexists with the traditional allocation mechanisms over the satellite link, but it does not replace them. This is particularly important because the allocations of OHRM can fail. This happens if the MN receives the satellite resources too early (in the case that the threshold of the manager, $P^{m n g r}$, is not correctly estimated) or if the link alerts to the NCC are lost. In both cases, the MN will not get uplink resources after the up handover. To cope with these situations, the manager changes the mode of the $\mathrm{MN}$ from virtual to satellite (and reallocates its resources) if the NCC receives an R/VBDC request (with non-null rate or volume) from the satellite terminal. This scenario is illustrated in Section 6.1.

4.3.2. Reactivation of the satellite allocation loop. After a down handover, the OHRM switch redirects the data flows to the terrestrial network. This stops the generation of the dynamic bandwidth requests (RBDC and VBDC) and the allocation loop without the intervention of the OHRM manager. When the MN returns to the satellite network, the allocation loop needs to be restarted. The restart is particularly slow in satcom because the propagation delay is very large. The reallocation of dynamic resources (RBDC and VBDC) is actually twice slower than for static resources (CRA) because the $\mathrm{R} / \mathrm{VBDC}$ requests of the terminals must first travel through the satellite link to reach the NCC. To speed up the restart of the loop, dynamic resources are artificially delivered with the static allocations. To do so, the NCC internally generates a dynamic RBDC shot. The required amount of resources $B^{\text {shot }}$ is estimated from the current data rate of the terrestrial link, $B_{\text {terlink }}$, as defined in equation 4 :

$$
B^{\text {shot }}=B_{\text {terlink }}-B^{C R A}
$$

where $B^{C R A}$ is the constant rate assigned to the MN. The OHRM manager obtains $B_{\text {terlink }}$ by sending

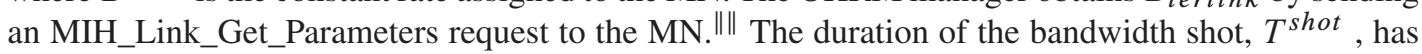
to be long enough to cover the needs of the terminal during the restart of the allocation loop. If the first R/VBDC request of the $\mathrm{MN}$ is generated $T_{\text {next }}^{r e q}$ seconds after the reallocation, the minimal value of $T^{\text {shot }}$ is

$$
T_{\text {min }}^{\text {shot }}=T_{\text {next }}^{r e q}+T_{\text {uplink }}^{\text {SAT }}
$$

\section{IMPLEMENTATION AND MODEL}

\subsection{Implementation}

Optimized handover and resource management has been implemented in the NS-2 network simulator. Our implementation is based on the MIH framework from the National Institute of Standards and Technology (NIST) (which includes NS-2.29) and the multi-frequency, time division multiple access (MF-TDMA) DAMA model from Aberdeen University. The satellite models of NS-2 have been updated with hierarchical addressing to make them compatible with the NIST framework.*** The MIH middleware has also been extended to support communications with MIH non-PoA entities such as the NCC. ${ }^{\dagger \dagger}$ The main features of our implementation are summarized in the succeeding text:

\footnotetext{
${ }^{\text {Tा TT }}$ The NCC must provision enough resources in the satellite network to guarantee the restoration of the resources of the virtual terminals.

\|\| or to the PoA if monitoring has been delegated.

$* * *$ The original satellite models only support flat addressing.

${ }^{\dagger \dagger}$ The NIST model only supports communications within the access network.
} 


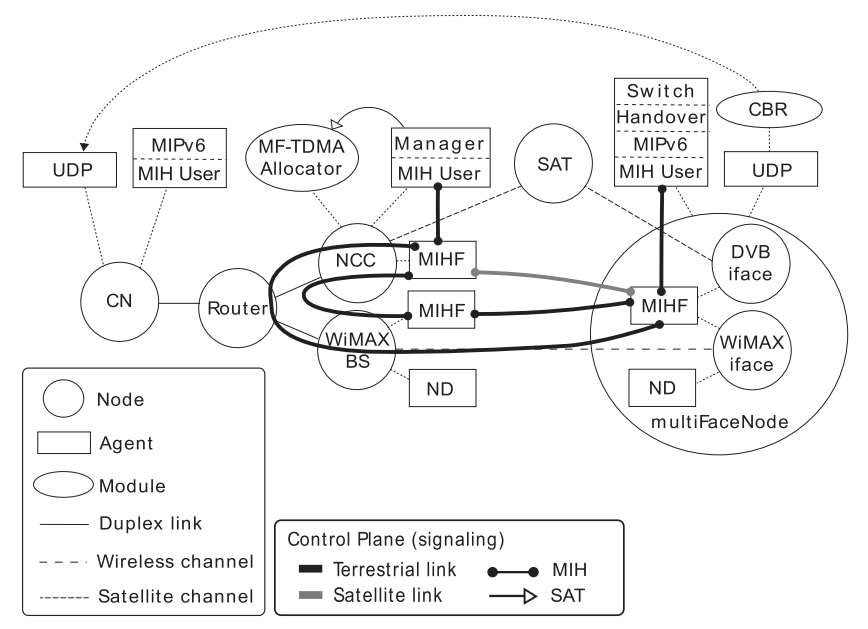

Figure 6. Simulation model.

- Application level

- Dynamic management of the resources of the NCC during handovers;

- MIH middleware

- Support of MIH communications with non PoA entities;

- Support of the HO_Complete commands in the MICS service;

- Support of remote MIH_Link_Parameters_Report events in the MIES service.

- Network level

- Basic support of MIH in the satellite interfaces;

- Integration of DVB-RCS(2);

- Support of hierarchical routing in the satellite models;

- Support of WiMAX operations over fading channels; $\$ \S \S$

- Packet transfer between NIC queues.

\subsection{Modeling}

Our simulation model is depicted in Figure 6. The MN is modeled with a multiface node [19]. It includes a DVB-RCS(2) [17, 20] and a WiMAX [21] NIC. The gateway in the DVB-RCS ground network is collocated with the resource allocator in the NCC. The satellite and the WiMAX segments belong to two different IP networks. The satellite NIC has a permanent IP address. According to the NS-2.29 implementation, the address of the WiMAX NIC is configured dynamically with the neighbor discovery (ND) protocol [19]: the MN requests the WiMAX network prefix when the link goes up. The ND agent in the WiMAX PoA broadcasts the prefix information in the router advertisement messages. A constant bit rate (CBR) flow is established between the $\mathrm{MN}$ and a corresponding node (CN) in the Internet. When the flow needs to be redirected, the MIPv6 agent sends a message to the $\mathrm{CN}$ to inform him of the new address to use [19]. The OHRM components are represented in bold. The MIHF function is installed in the MN, the PoA, and the NCC. The MIH signaling flows between the three nodes. The satellite signaling controls the release and restoration of the resources in the MF-TDMA DAMA allocator. The OHRM switch redirects the CBR flow and manages the transfer of the packets between the buffers of the NICs during handovers.

\footnotetext{
$\$+$ OHRM does not require the satellite NIC to support MIH. The basic primitives of MIES and MICS (MIH_Link_Up/Down, MIH_Link_Capability_Discover and MIH_Link_Event_(Un)subscribe) have only been added to simplify the access to the NIC. $\S \S$ The NIST model is not designed to support random packet losses. For instance, if the Dynamic Service Addition (DSA) exchange fails, the DSA packets are not retransmitted and so the link will not be usable. Our extension guarantees that a data connection can be established before the link is up.
} 
Table I. Propagation and mobility model parameters.

\begin{tabular}{|c|c|}
\hline Parameter & Value \\
\hline \multicolumn{2}{|c|}{ WiMAX } \\
\hline$\beta$ & 2 \\
\hline$P_{r}\left(d_{0}\right)($ Watt $)$ & $2.335 \mathrm{e}-8$ \\
\hline$P_{\text {reception }}$ (Watt) & $2.025 \mathrm{e}-12$ \\
\hline$P^{\text {switch }}$ (Watt) & $1.05 \times P_{\text {reception }}$ \\
\hline \multirow{2}{*}{\multicolumn{2}{|c|}{ DVB-RCS }} \\
\hline & \\
\hline \multicolumn{2}{|c|}{ Mobility } \\
\hline Estimate of speed $v(\mathrm{~m} / \mathrm{s})$ & 38 \\
\hline Estimate of angle $\alpha$ (rad) & $\pi / 2$ \\
\hline
\end{tabular}

DVB-RCS, digital video broadcasting return channel via satellite.

The radio propagation in the WiMAX cell follows the shadowing model [22]. The path loss at a distance $d$ of the WiMAX PoA is expressed with respect to some reference distance $d_{0}$ (here $1 \mathrm{~m}$ ) as

$$
\left[\frac{P_{r}(d)}{P_{r}\left(d_{0}\right)}\right]_{d B}=-10 \beta \log \left(\frac{d}{d_{0}}\right)+X_{\sigma}
$$

$\mathrm{X}$ is a random variable that models the shadowing effect. It has a Gaussian distribution with a zero mean and a standard deviation of $\sigma \mathrm{dB} . \beta$ is the path loss exponent. The low part of the simulation model is actually similar to [18], but our MIH implementation uses an exponential moving average of parameter 0.5 instead of a simple mean to average the signal power level in the radios. The MN starts in the satellite network and then moves across the WiMAX cell. The node estimates that at the exit of the WiMAX network, its velocity vector will be perpendicular to the tangent of the cell edge and that its speed $v$ will equal $38 \mathrm{~m} / \mathrm{s}$. $P^{m n g r}$ is computed from equation 3 . Assuming the inputs of Table I, the MN gets $P^{m n g r}=1.1 \times P_{\text {reception }}$.

The main parameters of the network are presented in Table II. We limit the validity of the network prefix to $60 \mathrm{~s}$ to better visualize the impact of channel fading on the terrestrial connection time. When the prefix expires, the MN sends a new router solicitation to the WiMAX PoA. The data queues are deliberately oversized to avoid packet loss during the transfer of the buffers between the WiMAX and DVB-RCS NICs.

The parameters of MIH and OHRM are shown in Table III. The MIH commands that are not confirmed are retransmitted. The bandwidth shot of OHRM supersedes the first traffic request of the MN after the reallocation. "TIT Most of the MIH and network parameters of the NIST mobility package have been kept unchanged. The differences are marked in italic in Tables II and III.

The CBR flow generates a packet of 160 bytes every $0.02 \mathrm{~s}$. It models a 64 kbps audio stream sent over the Internet.

\section{PERFORMANCE RESULTS}

\subsection{Packet delay}

The first four figures show the impact of handovers on the packet delay when the link is monitored by the MN. The node estimates its speed with a different error in the four scenarios. Figure 7 shows an

\footnotetext{
Tाबा This is because the interval of time until the next traffic request may be too short for the MN to correctly compute the $\mathrm{R} / \mathrm{VBDC}$ requests. Indeed, in the original MF-TDMA DAMA model, the volume and the rate of the requests are computed over one second.
} 
Table II. Network configuration.

\begin{tabular}{ll}
\hline Parameter & Value \\
\hline \multicolumn{1}{c}{ WiMAX } & \\
(PHY) Physical model & OFDM \\
(PHY) Modulation & BPSK \\
(PHY) Cyclic Prefix & 0 \\
(PHY) Frequency (GHz) & 3.5 \\
(PHY) Bandwidth (MHz) & 5 \\
(MAC) Data queue size (packets) & 500 \\
(MAC) Frame duration (s) & 0.004 \\
(MAC) DCD/UCD interval (s) & 5 \\
(MAC) Lost DL/UL_MAP interval (s) & 0.6 \\
(ND) Max \# of router sollicitations & 10 \\
(ND) Delay between retries (s) & 0.1 \\
(ND) validity of the network prefix (s) & 60 \\
& \\
& \\
(MAC) Data queue size (packets) & 500 \\
(MAC) Frame duration (s) & 0.0265 \\
(MAC) \# of frequency carriers per frame & 1 \\
(MAC) \# of timeslots per carrier & 16 \\
(MAC) \# of ATM cells per timeslot & 1 \\
(Traffic) RBDC enabled & yes \\
(Traffic) VBDC enabled & no \\
(Traffic) Traffic request interarrival & 1 \\
(Traffic) \# of CRA slots allocated per node per frame & 1
\end{tabular}

BPSK, binary phase-shift keying; DCD, downlink channel descriptor; UCD, uplink channel descriptor; DVB-RCS, digital video broadcasting return channel via satellite; CRA, constant rate assignment.

Table III. Media independent handover and optimized handover and resource management configuration.

\begin{tabular}{lll}
\hline Parameter & \multicolumn{1}{c}{ Value } \\
\hline & MIH & 0.5 \\
Command timeout (s) & & 3 \\
Max \# of retries & OHRM & 1 \\
& Up to the $2^{\text {nd }}$ next traffic request received \\
Link down timeout (s) & & \\
$T_{\text {shot }}$ &
\end{tabular}

MIH, media independent handover; OHRM, optimized handover and resource management.

example for which the actual value and the estimate of the speed are close. The DVB-RCS interface of the MN is turned on at time 0 . The CBR flow starts at the same time. The delay of the first packets increases because the NCC does not grant the MN enough CRA slots to carry the flow. The R/VBDC traffic requests are sent every second. The first request is sent at time 1 and it takes $0.3 \mathrm{~s}$ to reach the NCC. The RBDC resources allocated by the NCC are available at the MN side one propagation delay after. All in all, the MN receives its RBDC slots at time 1.6 s. The 12th CBR packet is generated at time $0.24=0.02 \tilde{\mathrm{A}} \mathrm{U}_{12}$ and experiences the maximum delay $(1.66 \mathrm{~s})$, which splits up into $1.36=1.6-0.24 s$ of waiting time in the NIC queue and $0.3 \mathrm{~s}$ of propagation time. The RBDC slots allow the MN to drain its buffers. After packet 230, there is no more waiting in the queue. The packet delay just accounts for the propagation time over the satellite link. The down handover happens at packet 500. The low delay is characteristic of WiMAX. The MN returns to the satellite network when 


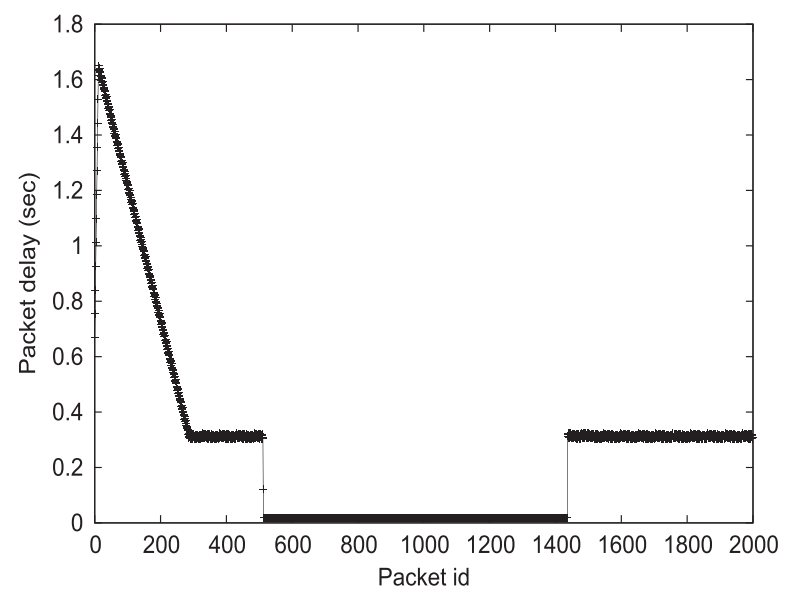

Figure 7. Optimized handover and resource management allocation for a speed of $30 \mathrm{~m} / \mathrm{s}$.

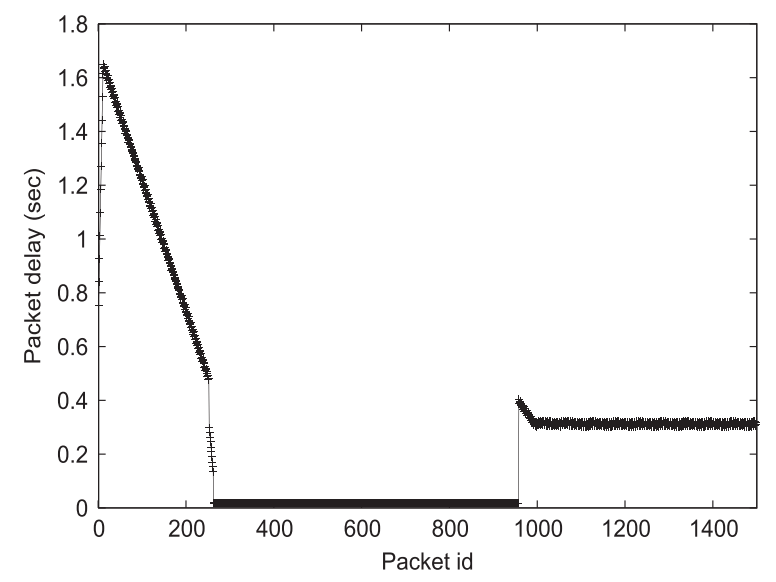

Figure 8. Optimized handover and resource management allocation for a speed of $45 \mathrm{~m} / \mathrm{s}$.

the power level of the signal falls below $P^{\text {switch }}$. The up handover occurs at packet 1400 . There is no handover delay because $P^{m n g r}$ is correctly estimated, \|\|\|\| and so the satellite slots are directly available when the traffic switches to the DVB-RCS interface.

In the second example (Figure 8), the speed of the MN is higher than the estimate. As a result, the satellite resources are not yet available when the traffic is redirected to the DVB-RCS interface. In this example, the handover occurs $70 \mathrm{~ms}$ too early, and so the packets have to wait an additional $70 \mathrm{~ms}$ delay to access the channel.

In the third example (Figure 9), the $\mathrm{MN}$ is too slow and arrives when the resources allocated by the NCC are about to expire. The DVB-RCS interface only receives a small part of the total allocation. The available resources only allows the MN to send the MIH_Link_Down indication and 19 CBR packets. The NCC restores the MN resources upon the reception of the link down indication. The maximum waiting is for the 20th CBR packet. It has to wait $0.3 \times 2-0.02 \times 20=0.2 s$ before receiving its first slots.

The scenario with the slowest speed is represented in Figure 10. The delay between the restoration of the resources and the up handover is so large that all the slots granted by OHRM are lost. The MN

\footnotetext{
"IIII Actually, the speed of the MN is underestimated by $25 \%=(38-30) / 30$, but the up handover can tolerate delay because the duration of the bandwidth shot is over-sized $\left(T^{\text {shot }} \gg T_{\text {min }}^{\text {shot }}\right)$.
} 


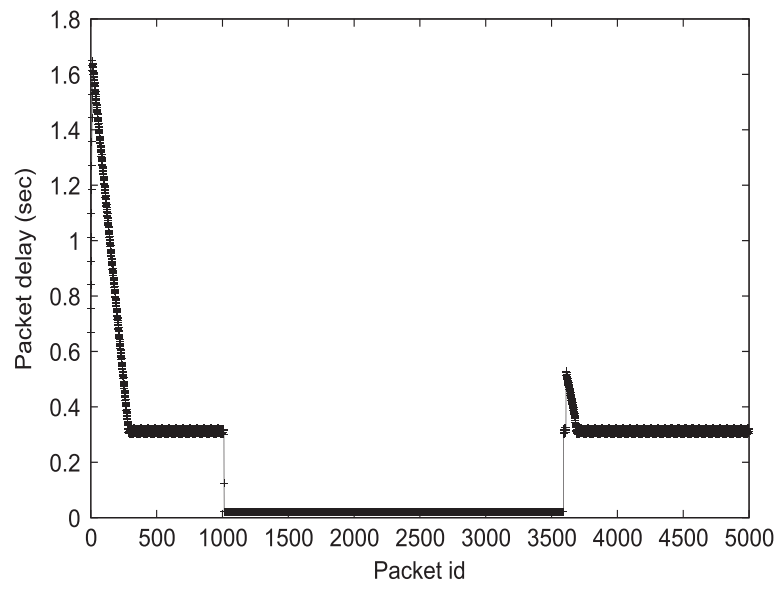

Figure 9. Optimized handover and resource management allocation for a speed of $12 \mathrm{~m} / \mathrm{s}$.

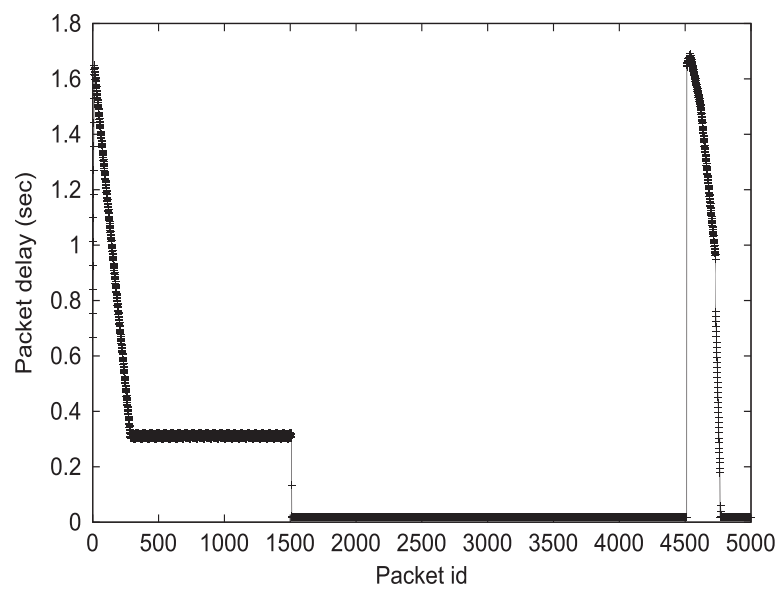

Figure 10. Allocation for a speed of $7 \mathrm{~m} / \mathrm{s}$.

has no way but to use the default DVB-RCS resources reservation procedure to access the channel in this case. The handover latency accounts for the time to restart the complete allocation loop.

The results of the previous scenarios are summarized in Figure 11. The extra delay induced by the up handover can be plotted either as a function of the speed of the MN or of the anticipation error $E_{a n t}$. $E_{a n t}$ is measured as the time difference between the actual time of the handover and its estimate. When the MN overestimates its speed $\left(E_{a n t}<-0.8\right)$, the handover comes too late and so the traditional channel access procedure is used. This is the scenario described in Figure 10. The lower bound of the extra delay in this case corresponds the minimum time required to restart the satellite allocation loop, that is, $0.6 s\left(=T_{\text {downlink }}^{S A T}+T_{\text {uplink }}^{S A T}\right)$. In our implementation, the traffic request interval is very large, which negatively impacts the packet delay. The performance can be improved (by decreasing the traffic request interval for instance) but within the limits of $0.6 \mathrm{~s}$. For small anticipation errors ( $E_{\text {ant }}$ varying from -0.4 to 0 ), the handover delay is nil. OHRM tolerates errors in the estimation of the speed of the MNs because the duration of the bandwidth shot is oversized ( $T^{\text {shot }} \gg T_{\text {min }}^{\text {shot }}$ ). However, premature handovers $\left(E_{a n t}>0\right)$ are penalized. The maximum delay $(0.6 \mathrm{~s})$ would be obtained for infinite speed. In this case, the trigger of the switch threshold and the redirection of the flows would occur at the same time. OHRM anticipation system would be short-cut, and the MN would have to wait $T_{\text {downlink }}^{S A T}+T_{\text {uplink }}^{S A T}(=0.6 \mathrm{~s})$ to get its slots. 


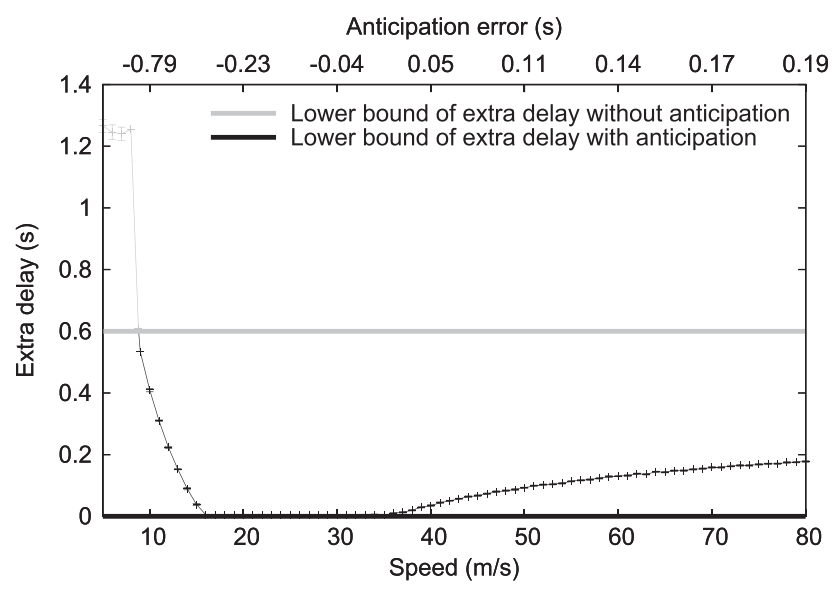

Figure 11. Extra delay induced by up handovers.

\subsection{Bandwidth usage and connection time}

We now show the benefits of the rollback feature and the delegation of monitoring. The distance, $d$, between the MN and the PoA is fixed and controlled by the mean power factor (MPF) of the WiMAX signal. The bandwidth losses and the duration of the WiMAX connection are studied for different fast fading conditions. The performance parameter is the standard deviation of the propagation model $\sigma$, which varies from 0 to $3 \mathrm{~dB}$. Figures 12 to 14 compare the performance with and without rollback event (Section 4.1.1). The link is monitored by the MN. Figure 12 shows the WiMAX bandwidth consumed by the link alerts to report the status of the terrestrial connection to the NCC. When the MPF is large (1.6), $\sigma$ has almost no effect on the MIH overhead. This is because the mean power is high and rarely crosses the manager threshold, $P^{m n g r}$. The impact of $\sigma$ is visible for lower MPFs. For MPF $=1.3$, the maximum overhead is obtained for $\sigma=0.8$. The overhead is twice larger when the roll back event is enabled, because every link alert is followed by a roll back, which basically doubles the control traffic. We show that for a given distance, $d$, the overhead is proportional to the probability $\operatorname{Prob}\left(P^{\text {switch }}<P_{r}(d, \sigma)<P^{m n g r}\right)$ that the power level of the packets is within $\left[P^{\text {switch }}, P^{m n g r}\right]$.

Figure 13 shows the waste of satellite resources in function of $\sigma$. The shape of the bell is proportional to the bandwidth loss in the terrestrial network but with a higher magnitude. The maximum losses are obtained when the rollback feature is disabled. They respectively reach 2.5 and $60 \mathrm{kbps}$ for MPF $=1.3$

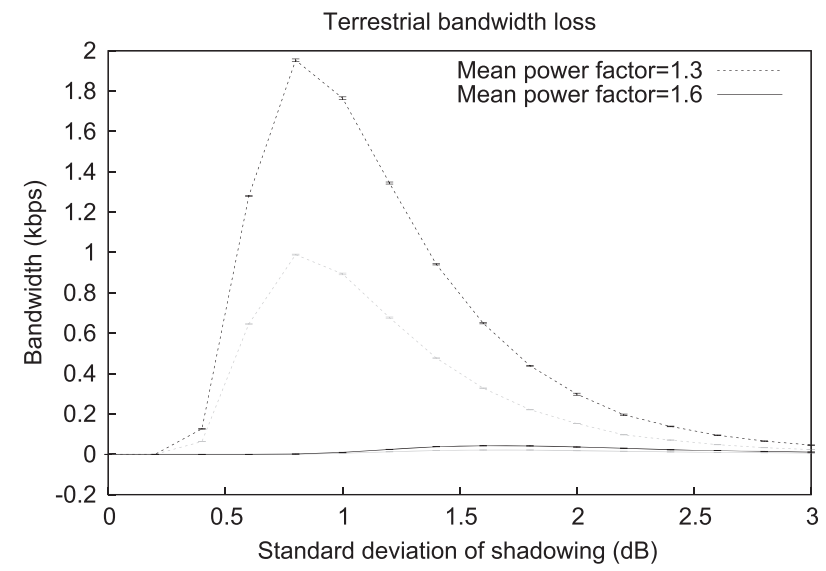

Figure 12. Terrestrial bandwidth loss with (black) and without (gray) rollback. Link monitored by the mobile node. 


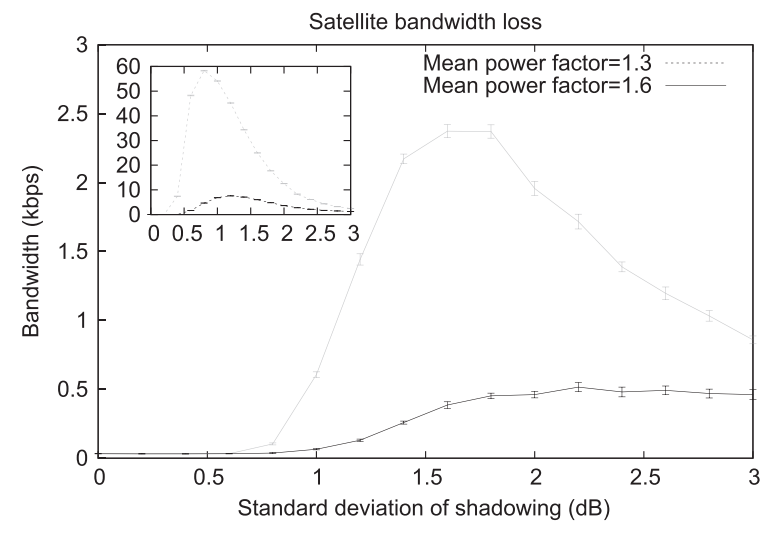

Figure 13. Satellite bandwidth loss with (black) and without (gray) rollback. Link monitored by the mobile node.

and 1.6. The performance is lower when rollback is disabled because the NCC does not release the resources until the link down timeout.

The duration of the WiMAX connection is plotted in Figure 14. The stay in the WiMAX network is limited to $60 \mathrm{~s}$, which explains the truncation of the curves. The connection time decreases when the MN gets closer to the border of the cell $(\mathrm{MPF} \rightarrow 1)$. The minimum duration is obtained when $\operatorname{Prob}\left(P^{\text {reception }}<\operatorname{Pr}(d, \sigma)<P^{\text {switch }}\right)$ is maximum. For MPF $=1.3$, this value is reached for $\sigma=1$. The results are the same with and without rollback because the duration of the connection only depends on the packet arrival rate and on the distribution of the signal power at the MN. Figures 15-17 show the performance when the link is monitored by the PoA. In the previous experiments, the monitoring was based on the WiMAX DL and UL MAPs received by the MN. Here, the PoA only receives data traffic from the MN, and the rate of this flow is 10 times lower than

the control traffic of the PoA. So, to allow comparison with the previous results, experiments with an equivalent traffic load have been added. The corresponding results are represented in gray in the figures. The rollback feature is enabled in all scenarios. The bandwidth loss in the terrestrial network is represented in Figure 15. The MIH overhead accounts for the link alerts sent before and after the delegation. It increases with the CBR rate and the distance with the PoA (low MPF) because the probability to generate an alert is higher in these cases. Still, the losses are very low in the four scenarios. These results confirm that PoAs are much more efficient than MNs to monitor links.

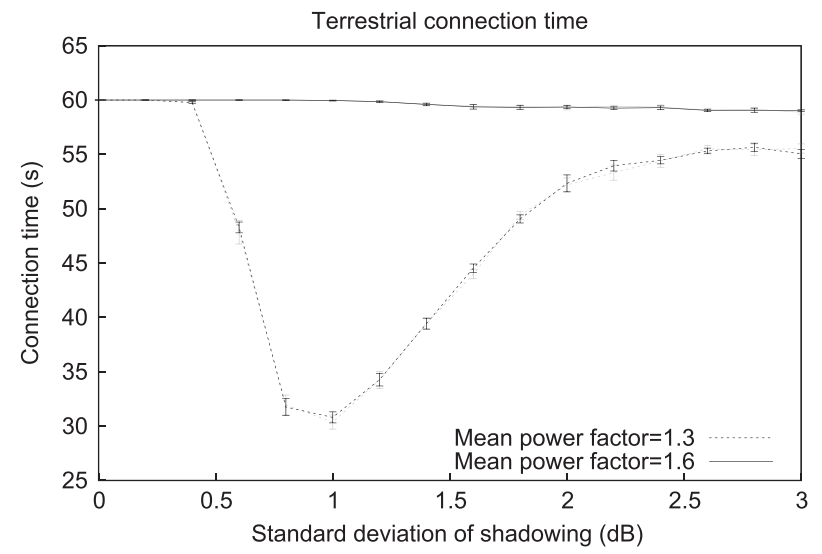

Figure 14. Terrestrial connection time with (black) and without (gray) rollback. Link monitored by the mobile node. 


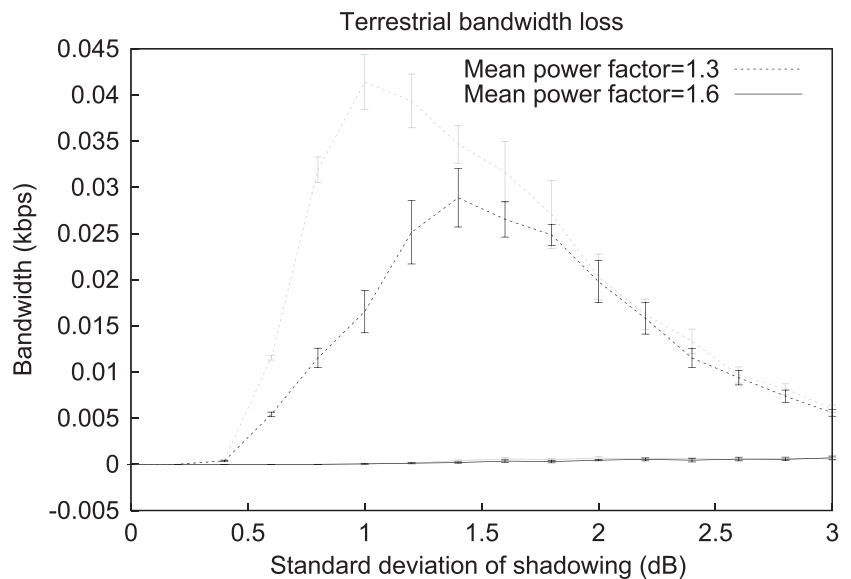

Figure 15. Terrestrial bandwidth loss. Low (black) and high (gray) load. Link monitored by the point of attachment.

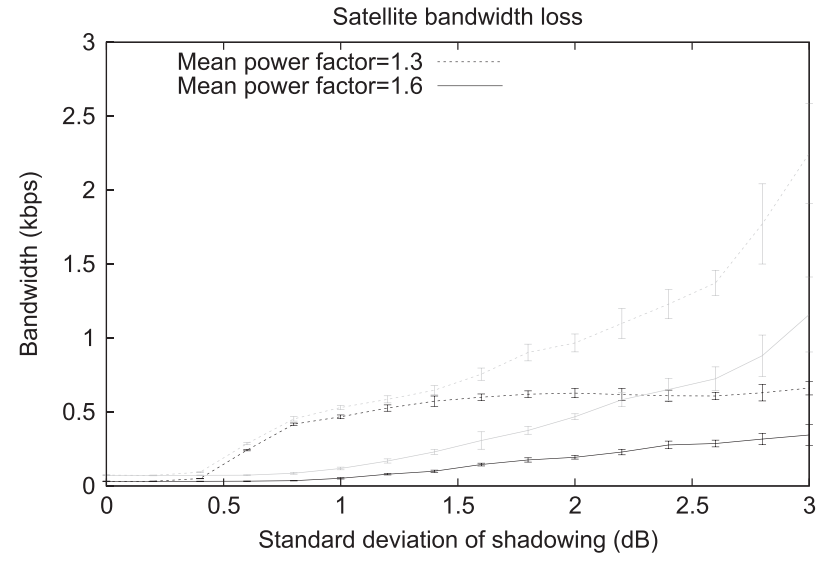

Figure 16. Satellite bandwidth loss. Low (black) and high (gray) load. Link monitored by the point of attachment.

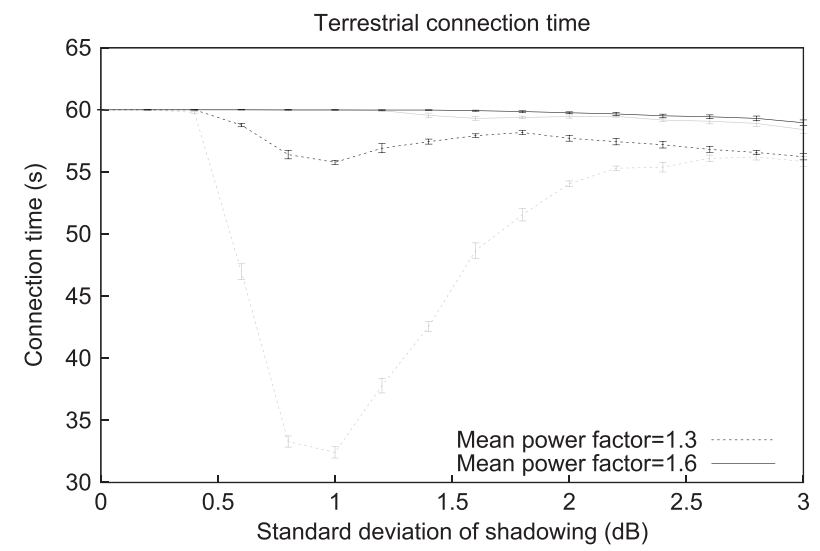

Figure 17. Terrestrial connection time. Low (black) and high (gray) load. Link monitored by the point of attachment. 
Figure 16 shows the satellite bandwidth loss due to fast fading. The loss increases with sigma but always remains below $2 \mathrm{kbps}$. This result has to be compared with the 10 and $60 \mathrm{kbps}$ lost when the link is monitored by the MN.

At same packet rate, the duration of the terrestrial connection does not change whether monitoring is carried out by the PoA or the MN. This is clearly shown by comparing the connection time of Figure 17 with the one of Figure 14.

\section{DISCUSSION}

From our experience with OHRM, we identified several issues regarding the applicability of MIH to real networks. One problem with WiMAX is that the MIH link up is triggered before the data connection is established with a digital signature algorithm exchange. As a consequence, the MIH applications have to make sure that the terrestrial link is really usable before redirecting the data flows.

The second issue concerns the support of MIH in the network equipment. The way the radio signal is averaged may have in this respect an important impact on the results. The size of the interval used to compute the data rate can also affect the performance. The basic rule is that the system must always be able to quickly estimate the rate of the flows after a handover.

Our third remark concerns reliability. MIH proposes an optional acknowledgement service for the MIH signaling when the underlying transport protocol is not reliable. Namely, over a WiMAX (Hybrid) automatic repeat request (ARQ) channel, we would recommend to deactivate the MIH acknowledgement service. The limitations of this service are the duration of the timeouts, which is too large ( $1 \mathrm{~s}$ ) in the context of $4 \mathrm{G}$, and the lack of support of simultaneous transactions (event or command) with a given peer. These constraints are relaxed in the MIH model of the NIST, which uses sub-second timers for retransmissions (Table III). We believe that, in a real network, this issue would be resolved at the transport layer. Transmission control protocol (TCP) would represent in our opinion the best transport for MIH as it is widely supported and reacts quickly to packet loss. Because our work has been performed with the assumption of seamless handover, no interaction will occur between TCP and MIH ARQ. However, WiMAX ARQ and TCP may interact, but transport is not the topic of this paper. Some studies can be found on this issue, in particular the use of cross layer schemes for TCP performance improvement [23].

Concerning a real deployment of the proposal, coordination between the two networks is ensured by the centralization of messages and decisions at the OHRM manager. Nevertheless, the real implementation of OHRM would encounter an issue if the system latency would be too important comparing with the optimization mechanism thresholds. This study has shown through simulation that the OHRM mechanism optimizes delay and resource use. However, these results are linked with the threshold parameters, which could be quite different in a real environment. The study has shown a first step to address that it consists in introducing OHRM in our test-bed to study the impact of the threshold on the ping-pong issue.

\section{CONCLUSION}

This paper has presented OHRM a scheme that optimizes handover and resource management in HSTNs. OHRM leverages on the MIH capabilities of the access network to shorten the signaling path to the satellite ground network. The use of the terrestrial network increases the system reactivity while saving capacity on the satellite side. An important point is that our solution does not require any modification in the satellite terminal interfaces. The simulation results show that the extra delay induced by up handovers can be completely removed if the restart of the satellite allocation loop is correctly anticipated. They also show the benefit of canceling the restart of the loop if the terrestrial link rolls back. In future works, we will propose new radio resource management algorithms that take into account the bandwidth gain of OHRM to increase the capacity of the satellite network. 
Another important issue is the synchronization between the allocation of resources in the NCC and the redirection of the flows in the mobile node. Ideally, the up handover should be perfectly synchronized with the reception of the satellite bursts. Finally, we plan to apply OHRM to other terrestrial technologies and satellite systems. A test-bed implementation, in the continuity of [8], will be an important step towards real deployment.

\section{REFERENCES}

1. IEEE. IEEE 802.21, Part 21: Media Independent Handover Services. IEEE Std 802.21, 2009.

2. Kota S, Giambene G, Kim S. Satellite component of NGN: integrated and hybrid networks, International Journal of Satellite Communications and Networking 2011; 29(3):191-208.

3. Evans B, Werner M, Lutz E, Bousquet M, Corazza G E, Maral G, Rumeau R. Integration of satellite and terrestrial systems in future multimedia communications, Wireless Communications 2005; 12(5):72-80.

4. ETSI. Satellite Earth Stations Systems (SES); Classication and Terminology of Systems Combining Satellite and Terrestrial Communication Systems. ETSI TR Draft, 2011.

5. ISI. Integrated Satellite Initiative European Technology Platform Strategic Research and Innovation Agenda-Edition 2011, 2011.

6. ITU-R. Recommandation of ITU-R M.1182, Integration of Terrestrial and Sattelite Mobile Communication Systems, 1995.

7. Klein-Lebbink E. Advanced satellite technology needs, IEEE/MTT-S International Microwave Symposium: Baltimore, MD, $2011 ; 1518-1522$.

8. Arnal F, Dhaou R, Fasson J, Bernard J, Barvaux D, Dubois E, Gélard P. Handover Management for Hybrid Satellite/Terrestrial Networks, Personal Satellite Services Conference (PSATS): Bradford, 2012; 95-103.

9. Piri E, Pentikousis K. Towards a GNU/Linux IEEE 802.21 Implementation, IEEE International Conference on Communications (ICC): Dresden, 2009; 1521-1525.

10. Corujo D, Guimaraes C, Santos B, Aguiar R L. Using an open-source IEEE 802.21 implementation for network-based localized mobility management, IEEE Communications Magazine 2011; 49(9):114-123.

11. Rouil R, Golmie N, Montavont N. Media independent handover transport using cross-layer optimized stream control transmission protocol, Computer Communications 2010; 33(9):1075-1085.

12. Buburuzan T, May G, Melia T, Modeker J, Wetterwald M. Integration of Broadcast Technologies with Heterogeneous Networks-An IEEE 802.21 Centric Approach, IEEE International Conference on Consumer Electronics (ICCE): Las Vegas, 2007; 1-2.

13. Salhani M, Dhaou R, Beylot A L. Terrestrial Wireless Networks and Satellite Systems Convergence, AIAA International Communications Satellite Systems Conference (ICSSC): Seoul, 2007; 1-5.

14. Qureshi R, Dadej A. Adding Support for Satellite Interfaces to 802.21 Media Independent Handover, IEEE International Conference on Networks (ICON): Adelaide, Australia, 2007; 542-546.

15. Fun $\mathrm{Hu}$ Y, Pillai P, Berioli M. Mobility Extension for Broadband Satellite Multimedia, International Workshop on Satellite and Space Communications (IWSSC): Siena-Tuscany, Italy, 2009; 62-66.

16. Freedman J, Rubin P, Kaplan T, Halvorson E, Eisenman A, Edalat F, Berman A, Markley S. Global Resource Manager for Mobile Satellite Systems Employing Non Uniform Frequency Allocations, AIAA International Communications Satellite Systems Conference: Anaheim, 2010; 331-336.

17. ETSI. Digital Video Broadcasting (DVB); Interaction Channel for Satellite Distribution Systems. ETSI EN 301790 V1.5.1, 2009.

18. Yoo S J, Cypher D, Golmie N. Predictive link trigger mechanism for seamless handovers in heterogeneous wireless networks, Wireless Communications and Mobile Computing 2009; 9(5):685-703.

19. National Institute of Standards and Technology. NS-2 Mobility Package, Jul 2007. http://www.antd.nist.gov/ seamlessandsecure.shtml [accessed on: February 2011].

20. ETSI. Digital Video Broadcasting (DVB); DVB-RCS2 Higher Layer Satellite Specification. ETSI TS 101 545-3 V1.1.1, 2012.

21. IEEE. IEEE 802.16, Part 16: Air Interface for Broadband Wireless Access Systems. IEEE Std 802.16, Edition (R2009), 2004

22. Rappaport T S. Wireless Communications: Principles and Practice, 2 edn., Prentice Hall: Austin, Texas, 2002.

23. Giambene G, Marchi S, Kota S. Cross Layer Schemes for TCP Performance Improvement in HetNets for Hight-Speed Trains, IEEE Global Telecommunications Conference (GLOBECOM): Houston, Texas, 2011; 1-6. 


\section{AUTHORS' BIOGRAPHIES}

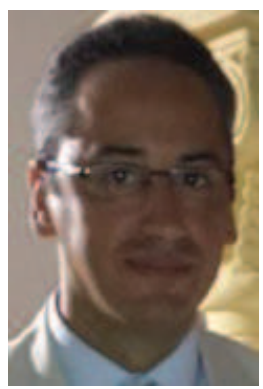

Riadh Dhaou is an associate professor at the Telecommunication and Networking Division of the INPT/ENSEEIHT (Institut National Polytechnique de Toulouse). Since September 2003, he is a member of the IRT team of the IRIT Lab (CNRS-UMR 5505) and of TéSA Lab. He received the 'Diplome d'Ingenieur Concepteur en Informatique' from the Ecole Nationale des Sciences de l'Informatique (ENSI), University of Tunis II in 1997, and the Diplome d'Etudes Approfondies (D.E.A.) in Computer Systems from the Université Pierre et Marie Curie in Paris (Paris VI) in 1998. In November 2002, he was awarded a PhD degree in Computer Systems, Telecommunication and Electronics by the University of Paris VI. His research interests include statistical characterisation and modelling of mobility, mobile and space communications, cross layer schemes modelling and optimisation, performance analysis of wireless networks, autonomous multi hop/cooperative communications systems, capacity and outage analysis of multi-user heterogeneous wireless systems, resource allocation, design and performance evaluation of wireless sensor networks, and energy consumption optimisation.

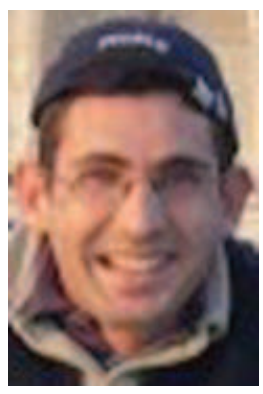

Ryad Ben-El-Kezadri received his Master's degree and $\mathrm{PhD}$ degree from the University Pierre and Marie Curie, Paris, France in 2001 and 2007, respectively. He was a research scientist at the University of California, Los Angeles (UCLA), Computer Science Department. Then, he was a research scientist at the IRIT/ENSEEIHT at the University of Toulouse. His research interests include wireless networks, network simulation and system design.

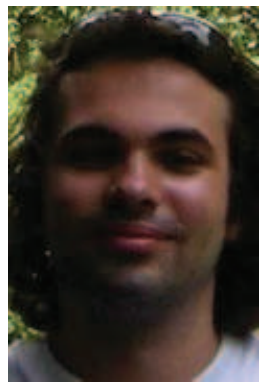

Julien Fasson has received the 'Diplôme d'Ingenieur en Construction Aéronautique' from the École Nationale Supérieure en Construction Aéronautique, Toulouse in 2001, and a PhD degree on Computer Systems and Telecommunications from the University of Toulouse in 2004. Since 2005, he is an assistant professor at the Telecommunication and Networking Division of the INPT/ENSEEIHT, and he is working at IRIT laboratory. His main interests are focused on the integration of satellite systems in terrestrial networks and the networking architecture for integrated service (P2P, content network, LTE, IMS, etc.).

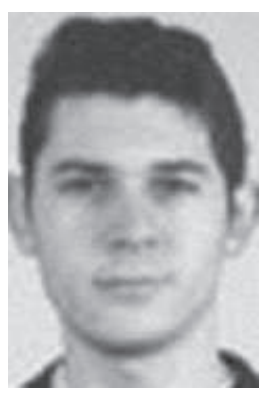

Fabrice Arnal is a network and access research engineer for Satellite Communications. $\mathrm{He}$ joined Thales Alenia Space in Toulouse in 2006. He holds a PhD dealing with reliability management for multicasting over satellite links. He has participated in several European Union projects such as SatSix and R\&T Centre National D'études Spatiales projects. His main topics of interest include cross-layering optimization, IP mobility services and handover management, transport layer, QoS and resource management.

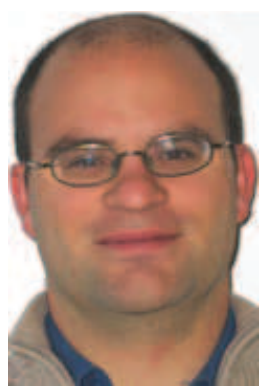

Emmanuel Dubois is a Network \& Telecom research engineer. He joined Centre National D'études Spatiales in Toulouse in 2008. He holds a PhD dealing with convergence in satellite networks. His main topics of interest include cross-layering optimization, IP mobility services and handover management, transport layer, QoS and resource management. 


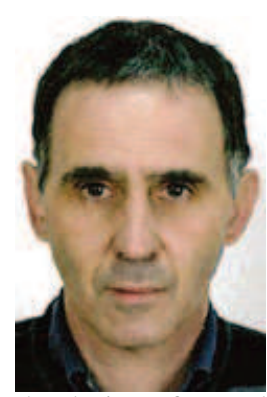

Patrick Gelard is a Network \& Telecom research engineer, expert senior at Centre National D'études Spatiales (CNES). He began his professional experience at Alcatel Bussiness System on private automatic branch exchange for 4 years before joining the CNES in 1990 as a protocol engineering expert at the Information Systems Division. Then, for 8 years, joined the CNES radio frequency divison to work on terrestrial and satellite networking. The main projects in telecommunications in which he was involved at CNES are in the development of an innovative 'IP-Phone' system design working on Ethernet local area network, the work on dedicated ATM infrastructure design for $50 \mathrm{Mb} / \mathrm{s}$ spacecraft telemetry real time delivery (named ATLAS), the development of a tool of remote teaching and E-learning based on 'Java Multimedia Framework' for hybrid context broadcast satellite/broadband terrestrial, the design of an operational 'heavy duty' (300 Gb/day) remote archiving system, the design of a worldwide (Marquise, Guyana) ground communication system for satellite telemetry monitoring and automated Massachusetts Institute of Technology alert, the development of an open multimedia digital video broadcasting-satellite experimental platform, and an expertise in satellite communications project at CNES (Agora, ATF, Athena-Fidus, MM2G, THD). He participated at satellite E-learning experiment over 250 French schools and was also involved in Internet proxying, caching, streaming, multicast and asymetric routing architectures. For the past 5 years, he is in charge of the research axis terrestrial and satellite infrastructure convergence. 